Not to appear in Nonlearned J., 45.

\title{
The Emission from Inner Disk and Corona in the Low and Intermediate Spectral States of Black Hole X-ray Binaries
}

\author{
Erlin Qiao $^{1}$ and B. F. Liu ${ }^{1}$ \\ National Astronomical Observatories, Chinese Academy of Sciences, Beijing 100012, China \\ qiaoel@nao.cas.cn
}

\begin{abstract}
Recent observations reveal that a cool disk may survive in the innermost stable circular orbit (ISCO) for some black hole X-ray binaries in the canonical low/hard state. The spectrum is characterized by a power law with a photon index $\Gamma \sim 1.5-2.1$ in the range of $2-10 \mathrm{keV}$ and a weak disk component with temperature of $\sim 0.2 \mathrm{keV}$. In this work, We revisit the formation of such a cool, optically thick, geometrically thin disk in the most inner region of black hole $\mathrm{X}$-ray binaries at the low/hard state within the context of disk accretion fed by condensation of hot corona. By taking into account the cooling process associated with both Compton and conductive processes in a corona, and the irradiation of the hot corona to the disk, we calculate the structure of the corona. For viscosity parameter $\alpha=0.2$, it's found that the inner disk can exist for accretion rate ranging from $\dot{M} \sim 0.006-0.03 \dot{M}_{\mathrm{Edd}}$, over which the electron temperatures of the corona are in the range of $1-5 \times 10^{9} \mathrm{~K}$ producing the hard X-ray emission. We calculate the emergent spectra of the inner disk and corona for different mass accretion rates. The effect of viscosity parameter $\alpha$ and albedo $a$ ( $a$ is defined as the energy ratio of reflected radiation from the surface of the thin disk to incident radiation upon it from the corona) to the emergent spectra are also presented. Our model is used to explain the recent observations of GX 339-4 and Cyg X-1, in which the thin disk may exist at ISCO region in the low/hard state at luminosity around a few percent of $L_{\text {Edd }}$. It's found that the observed maximal effective temperature of the thermal component and the hard X-ray photon index $\Gamma$ can be matched well by our model.
\end{abstract}

Subject headings: Accretion, accretion disks - Black hole physics - X-rays: individual (GX 339-4, Cyg X-1) - X-rays: stars

\footnotetext{
${ }^{1}$ National Astronomical Observatories, Chinese Academy of Sciences, Beijing 100012, China
} 


\section{Introduction}

Black hole X-ray binaries (BHXRBs) are binary star systems that are luminous in the $\mathrm{X}$-ray part of the spectrum. These X-ray emissions are generally thought to be caused by one of the component stars being a black hole accreting matter from the companion. The property of these systems have been reviewed by several authors (Remillard \& McClintock 2006; Done et al. 2007; Gilfanov et al. 2010). It is well-known that various spectral states have been exhibited in BHXRBs. In particular, two basic X-ray spectral states are presented with a high/soft spectral state occurring at high luminosities and a low/hard spectral state occurring at low luminosities. Generally, at the high/soft state, with higher accretion rate, the accretion is dominantly via a standard thin disk extending to the innermost stable circular orbit (ISCO) (Pringle \& Rees 1972; Shakura \& Sunyaev 1973; Mitsuda et al. 1984; Frank et el. 2002); The ISCO is at $3 R_{\mathrm{S}}$ (where $R_{\mathrm{S}}=2 G M / c^{2}$, G is gravitational constant, c is light speed, and $M$ is the central black hole mass) for non-rotating black holes and $0.615 R_{\mathrm{S}}$ for rotating black hole with limiting spin rate $a_{*}=0.9982$, where $a_{*}$ is specific angular momentum (Thorne 1974); With decline of the accretion rate to some transition rate, BHXRBs enter the low/hard spectral state, in which the standard thin disk is replaced by a hot, optically thin, geometrically thick advection dominated accretion flows (ADAF) in the most inner region around the black hole. i.e., the thin disk truncates at some radius off the ISCO (Rees et al. 1982; Narayan \& Yi 1994, 1995a, b; Abramowicz et al. 1995; Narayan 2005, 2008; Kato et al. 2008; Esin et al. 1997; Kawabata \& Mineshige 2010; Qiao et al. 2010; Zhang et al. 2010).

However, the picture of truncated outer thin disk + inner ADAF model in the low/hard state is challenged by the recent observations of some BHXRBs. Reis et al. (2010) investigate a sample composed of eight BHXRBs in the low/hard state observed by XMM-Newton and Suzaku. Although the hard X-ray continuum is characterized by a power law with a photon index in the range of $\Gamma \sim 1.5-2.1$, a thermal component with a color temperature consistent with $L \propto T^{4}$ is detected in all eight sources, meanwhile, broad iron $\mathrm{K}_{\alpha}$ fluorescence line is also observed in half of the sample. Both the $L \propto T^{4}$ relation and the broad iron line profile suggest that a cool disk extends to ISCO. The observed thermal component is around $0.2 \mathrm{keV}$. Observations to GX 339-4 are made by Swift and RXTE at 2007 May when the transition to the low/hard state occurs (Kalemci et al. 2007). Fits to the observed broad iron $\mathrm{K}_{\alpha}$ line profile with relativistic reflection model at luminosity $0.8 \% L_{\text {Edd }}$ suggests that a cool disk resides in the region very close to ISCO. A thermal component with a disk temperature $\sim 0.165 \mathrm{keV}$ at luminosity $0.8 \% L_{\mathrm{Edd}}$ is detected. The X-ray spectra are roughly characterized by a power law with a photon index $\Gamma=1.63_{-0.03}^{+0.04}$ (Tomsick et al. 2008).

A disk accretion model maintained by the recondensation of hot corona has been pro- 
posed to understand the presence of the cool disk in the most inner region around the black hole in the low/hard state by Liu et al. (2006), Meyer et al. (2007). In this model, when the accretion rate decreases just below the transition rate between high/soft state and low/hard state, as a consequence of efficient evaporation, the thin disk truncates at a distance where the maximum evaporation rate occurs (Meyer et al. 2000; Liu et al. 1999, 2002). The remnant disk from the truncation radius inwards can exist steadily fed by the condensation of the hot corona/ADAF rather than be swallowed by the black hole within the viscous time scale (Liu et al. 2007; Taam et al. 2008; Liu et al. 2011). The size of the inner remnant disk is governed by the accretion rate. The geometry of the accretion flows are allocated as an inner disk and a much cooler outer disk, which are separated by an ADAF. With decrease of the accretion rate, the inner disk shrinks and eventually vanishes completely at a certain accretion rate. The dynamical interaction between the inner disk and corona is studied by Liu et al. (2006), Meyer et al. (2007) in which only the vertical conductive cooling of the corona is considered throughout the corona. Furthermore, the Compton cooling of the corona by the soft photos from the underlying thin disk is taken into account. The cooling of the corona is classified as Compton-dominated case and conduction-dominated case at different radius. For Compton-dominated case, conductive cooling is neglected, and for conduction-dominated case, Compton cooling is neglected (Liu et al. 2007). However, for a certain mass accretion rate, Compton cooling and conductive cooling are comparable. In this case, both the Compton cooling and conductive cooling should be considered together. The cooling of the corona is modified by Taam et al. (2008), in which the effect of conductive cooling to the corona is added to Compton-dominated case, the effect of Compton cooling to the corona is added to conduction-dominated case.

In this paper, in order to get a radially continuous, self-consistent disk-corona model, we employ the result of Taam et al. (2008) and consider the irradiation of the hot corona to the disk (Liu et al. 2011). We calculate the structure of the corona and the emergent spectra of the inner disk and corona with different parameters of the model (In our calculation, contribution of the ADAF gas in the gap between the inner disk and outer disk, and the outer disk and corona to the spectra are not included). For the emission of inner disk, both the accretion luminosity contributed from disk accretion and the irradiation of the corona are included. Our model is used to explain the recent observations of black hole X-ray binaries GX 339-4 and Cyg X-1 in the low/hard spectral state. In section 2, the disk-corona model is briefly described. In Section 3, we show the numerical results of the structure and the emergent spectra of the inner disk-corona system. In Section 4, we compare the model predictions with observations. Our conclusions are presented in Section 5. 


\section{The model}

The disk-corona model adopted here, is based on the study of Taam et al. (2008) and Liu et al. (2011). It is assumed that an ADAF-like hot corona (described by the self-similar solution of Narayan \& Yi 1995, with $\alpha=0.2, \beta=0.8$ ) lies above a thin disk. The corona is heated by the viscous release of gravitational energy of accreted gas and cooled by vertical conduction and inverse Compton scattering of soft photons emitted by the underlying disk. For the Compton cooling of the corona, the upward hard photons escape from the corona directly; the downward hard photons are partially reflected and partially absorbed by the underlying optically thick disk. The absorbed photons are reprocessed in the optically thick disk and remitted as a thermal emission providing the soft photons for the Compton scattering in the corona. This procedure is iterated until a stable disk-corona system forms. In the vertical transition layer between the disk and corona, an equilibrium is established between the conductive flux from the upper corona, bremsstrahlung radiation, and vertical enthalpy flux. For a given distance from the black hole, a fraction of the disk gas is heated and evaporated to the corona when the conduction flux is too large to be radiated away. On the other hand, a certain amount of coronal gas is cooled down, condensing to the disk if the bremsstrahlung radiation is more efficient than the conduction. At accretion rates around a few percent of Eddington value, gas evaporates from the disk to the corona, the disk vanishes at around a few hundred Schwarzschild radii, and the coronal gas partially condenses back to the disk in the innermost region.

The cooling of the corona is classified as conduction-dominated case and Comptondominated case at different radius (Liu et al. 2007). Here, we follow the work of Taam et al. (2008) in which the conduction-dominated case is modified by adding the Compton cooling, and the Compton-dominated case is modified by adding the conductive cooling. Meanwhile, in our calculation the irradiation of the corona to disk is added for getting a self-consistent disk-corona system (Liu et al. 2011). Throughout the paper, we scale some quantities as, $m$ is the central black hole mass scaled with solar mass $M_{\odot} . \dot{m}$ is the accretion rate scaled

with Eddington accretion rate $\dot{M}_{\text {Edd }}, \dot{M}_{\text {Edd }}=1.39 \times 10^{18} \mathrm{mg} \mathrm{s}^{-1} . r$ is the distance from the black hole scaled with Schwarzschild radius $R_{\mathrm{S}}, R_{\mathrm{S}}=2 G M / c^{2}=2.95 \times 10^{5} \mathrm{~m} \mathrm{~cm}$. For the sake of clarity, we list the basic results of the disk-corona model in the following.

\subsection{Corona Dominated by Conductive Cooling}

In the case of conduction dominated cooling the condensation rate is given as (Taam et al. 2008) 


$$
\begin{gathered}
\dot{m}_{\mathrm{cnd}}(x)=3.23 \times 10^{-3} \alpha^{-7} \dot{m}^{3} f(x), \\
f(x)=\left(\frac{r_{o}}{r_{1}}\right)^{3 / 5}\left[6\left(\frac{r_{1}}{r_{o}}\right)^{1 / 10}-6\left(\frac{r_{1}}{r_{o}}\right)^{1 / 10} x^{1 / 2}-3 \int_{r_{i} / r_{o}}^{1}(1+\lambda)^{-2 / 5} x^{-2 / 5} d x\right],
\end{gathered}
$$

where $\lambda=\frac{q_{\mathrm{Cmp}}}{d F_{\mathrm{c}} d z}$ is the ratio of Compton cooling rate to conductive cooling rate, which weighs the modification of neglected cooling process compared to the dominated cooling process. The ratio can be reexpressed as $\lambda=1.4052 \times 10^{4} m r^{-3 / 2}\left[1-\left(\frac{3}{r}\right)\right] T_{\text {eff,max }}^{4}$, where $T_{\text {eff,max }}$ is the maximum effective temperature of the accretion disk (Taam et al. 2008). $r_{1}$ and $r_{o}$ represent the size of the inner disk without and with modification in conduction-dominated case respectively. $r_{1}$ and $r_{o}$ are expressed as,

$$
\begin{aligned}
& r_{1}=0.815 \alpha^{-28 / 3} \dot{m}^{8 / 3} \\
& r_{o}=0.815 \alpha^{-28 / 3} \dot{m}^{8 / 3}\left[1+\lambda\left(r_{o}\right)\right]^{4} .
\end{aligned}
$$

The electron temperature distribution of the corona in radial direction is,

$$
T_{\text {ec }}=2.01 \times 10^{10} \alpha^{-2 / 5} \dot{m}^{2 / 5} r^{-2 / 5}(1+\lambda)^{-2 / 5} \mathrm{~K} .
$$

The Bremsstrahlung luminosity from the transition layer is

$$
\frac{L_{\text {brem }}}{L_{\text {Edd }}}=0.0642 \alpha^{-7 / 3} \dot{m}^{5 / 3}\left[1-\left(\frac{3}{r_{o}}\right)^{1 / 2}\right],
$$

and the Compton luminosity from the corona is

$$
\begin{gathered}
\frac{L_{\mathrm{Cmp}}}{L_{\mathrm{Edd}}}=1.349 \alpha^{-7 / 5} m \dot{m}^{7 / 5}\left(\frac{T_{\mathrm{eff}, \mathrm{max}}}{0.3 \mathrm{keV}}\right)^{4} \\
\times \int_{r_{i} / 3}^{r_{o} / 3}(1+\lambda)^{-2 / 5} x^{-29 / 10}\left(1-x^{-1 / 2}\right) d x .
\end{gathered}
$$

Note that the emission from the outer pure ADAF is not included here, it would cause the coronal luminosity deviating from the true value when the inner disk is very small, which occurs at accretion rates less than 0.01 . 


\subsection{Corona Dominated by Compton Cooling}

In the case of Compton dominated cooling the condensation rate is given as,

$$
\dot{m}_{\mathrm{cnd}}(x)=A\left\{2 B\left[\left(\frac{r_{o}}{r_{i}}\right)^{1 / 2}-1\right]-\int_{r_{i} / 3}^{r_{o} / 3}\left(1+\frac{1}{\lambda}\right)^{-2 / 5} x^{1 / 5}\left(1-x^{-1 / 2}\right)^{-2 / 5} d x\right\},
$$

where

$$
\begin{aligned}
& A=6.164 \times 10^{-3} \alpha^{-7 / 5} m^{-2 / 5} \dot{m}^{7 / 5}\left(\frac{T_{\text {eff,max }}}{0.3 \mathrm{keV}}\right)^{-8 / 5}, \\
& B=3.001 \alpha^{-14 / 15} m^{2 / 5} \dot{m}^{4 / 15}\left(\frac{T_{\text {eff,max }}}{0.3 \mathrm{keV}}\right)^{8 / 5}\left(\frac{r}{3}\right),
\end{aligned}
$$

and the condensation radius $r_{o}$ meets the following formula,

$$
\begin{array}{r}
r_{o}\left[1-\left(\frac{3}{r_{o}}\right)^{1 / 2}\right]^{-4 / 7}=14.417 \alpha^{-4 / 3} m^{4 / 7} \dot{m}^{8 / 21} \\
\left(\frac{T_{\text {eff }, \max }}{0.3 \mathrm{keV}}\right)^{16 / 7}\left(1+\frac{1}{\lambda\left(r_{o}\right)}\right)^{4 / 7}
\end{array}
$$

The electron temperature distribution of the corona in radial direction is,

$$
\begin{array}{r}
T_{\mathrm{em}}=3.025 \times 10^{9} \alpha^{-2 / 5} m^{-2 / 5} \dot{m}^{2 / 5} r^{1 / 5} \\
\times\left[1-\left(\frac{3}{r}\right)^{1 / 2}\right]^{-2 / 5}\left(1+\frac{1}{\lambda}\right)^{-2 / 5}\left(\frac{T_{\mathrm{eff}, \max }}{0.3 \mathrm{keV}}\right)^{-8 / 5} .
\end{array}
$$

The expression of Bremsstrahlung luminosity from the transition layer is same as equation (5), and the the Compton luminosity from the corona is,

$$
\begin{aligned}
& \frac{L_{\mathrm{Cmp}}}{L_{\mathrm{Edd}}}=0.392 \alpha^{-7 / 5} m^{3 / 5} \dot{m}^{7 / 5}\left(\frac{T_{\text {eff,max }}}{0.3 \mathrm{keV}}\right)^{12 / 5} \\
& \quad \times \int_{r_{i} / 3}^{r_{o} / 3}\left(1+\frac{1}{\lambda}\right)^{-2 / 5} x^{-23 / 10}\left(1-x^{-1 / 2}\right) d x .
\end{aligned}
$$

For given black hole mass $m$, accretion rate $\dot{m}$, viscosity parameter $\alpha$, and a presumed value of $T_{\text {eff,max }}$, quantities describing the inner disk and corona are determined by eqs. (1) to (6) in the case of conduction dominated cooling or by (7) to (11) in the case of Compton dominated cooling. Iterative calculations are carried out until a self-consistent $T_{\text {eff,max }}$ is obtained. 


\subsection{Iterative Calculations with Irradiation}

The surface effective temperature of the accretion disk with irradiation from the corona is expressed as,

$$
\sigma T_{\mathrm{eff}}^{4}(r)=F_{\mathrm{cnd}}+F_{\mathrm{irr}},
$$

where $F_{\text {cnd }}$ refers to the flux which originates from disk accretion fed by condensation in per unit area, i.e.,

$$
F_{\text {cnd }}=\frac{3 G M \dot{M}_{\mathrm{cnd}}}{8 \pi R^{3}}\left[1-\left(\frac{3 R_{\mathrm{S}}}{R}\right)^{1 / 2}\right],
$$

$F_{\text {irr }}$ refers to the the illumination flux from the corona to the disk surface per unit area (Liu et al. 2011),

$$
\begin{array}{r}
F_{\text {irr }}=\frac{1}{2} L_{\mathrm{c}, \text { in }}(1-a) \frac{H_{\mathrm{s}}}{4 \pi\left(R^{2}+H_{\mathrm{s}}^{2}\right)^{3 / 2}} \\
L_{\mathrm{c}, \text { in }}=L_{\mathrm{brem}}+L_{\mathrm{Cmp}},
\end{array}
$$

where the ADAF-like corona is assumed above the disk as a point source at a height $H_{\mathrm{s}}$, and the covering factor of the point source to a disk ring at distance $R$ is given as $f=\frac{H_{\mathrm{s}}}{4 \pi\left(R^{2}+H_{\mathrm{s}}^{2}\right)^{3 / 2}}$,

$a$ is albedo. $L_{\mathrm{c}, \text { in }}$ is the total intrinsic luminosity of the corona and the transition layer above and below the disk.

Combining eqs. (12)-(14) the effective temperature of the accretion disk can be reexpressed as,

$$
\begin{aligned}
T_{\text {eff }}(r) & =2.05 T_{\text {eff,max }}^{\prime}\left(\frac{3}{r}\right)^{3 / 4}\left[1-\left(\frac{3}{r}\right)^{1 / 2}\right]^{1 / 4} \\
& \times\left[\frac{1+6 L_{\mathrm{c}, \text { in }}(1-a)}{\dot{M}_{\mathrm{cnd}} c^{2}} \frac{H_{\mathrm{s}}}{3 R_{\mathrm{s}}}\right]^{1 / 4} \\
= & 2.05 T_{\text {eff, } \max }\left(\frac{3}{r}\right)^{3 / 4}\left[1-\left(\frac{3}{r}\right)^{1 / 2}\right]^{1 / 4},
\end{aligned}
$$

where

$$
T_{\text {eff,max }}=T_{\text {eff,max }}^{\prime}\left[\frac{1+6 L_{\mathrm{c}, \text { in }}(1-a)}{\dot{M}_{\mathrm{cnd}} c^{2}} \frac{H_{\mathrm{s}}}{3 R_{\mathrm{s}}}\right]^{1 / 4} .
$$

$T_{\text {eff,max }}^{\prime}$ refers to the maximum effective temperature from disk accretion which is reached at $r_{\text {tmax }}=(49 / 12)$. The expression of $T_{\text {eff,max }}^{\prime}$ is given as (Liu et al. 2007),

$$
T_{\text {eff,max }}^{\prime}=0.2046\left(\frac{m}{10}\right)^{-1 / 4}\left[\frac{\dot{m}_{\mathrm{cnd}\left(\mathrm{r}_{\text {tmax }}\right)}}{0.01}\right]^{1 / 4} \mathrm{keV}
$$


At lower accretion rate $\dot{m}$, it's assumed that the corona is dominated by conductive cooling. An effective temperature $T_{\text {eff,max }}$ is presumed to calculate the condensation rate from eqs. (1) and (21), the luminosity of the transition layer and the corona from eqs. (5) and (6), with which a new effective temperature $T_{\text {eff,max }}$ is derived from eqs. (16) and (17) by assuming a value of albedo $a$. An iteration is made till the presumed temperature is consistent with the derived value. From the derived effective temperature, the Comptondominated region is determined the following equation (Liu et al. 2007), which is,

$$
r_{\mathrm{cmp}}\left[1-\left(\frac{3}{r_{\mathrm{cmp}}}\right)^{1 / 2}\right]^{-2 / 3} \leq 23.487 m^{2 / 3}\left(\frac{T_{\mathrm{eff}, \mathrm{max}}}{0.3 \mathrm{keV}}\right)^{8 / 3}
$$

If eq. (18) has no solution, it means the corona is dominated by conductive cooling throughout the corona, we find a self-consistent solution of the disk-corona system. Otherwise, the Compton-dominated region is determined by eq. (18). We recalculate the condensation rate by combing eqs. (11), (2) and (7), (8), and the luminosity of transition layer and corona from eqs. (5), (6) and (11) till the presumed temperature is consistent with the derived value, we find a solution of disk-corona system. With increase of the mass accretion rate, Compton-dominated region extends inward and outward till Compton cooling dominates throughout the corona at some accretion rate. An effective temperature $T_{\text {eff,max }}$ is assumed to calculate the condensation rate from eqs. (7), (8), and the luminosity from eqs. (15) and (11), with which a new effective temperature $T_{\text {eff,max }}$ is calculated from eqs. (16) and (17). Iterations are made till the presumed temperature is consistent with the derived value, we find a solution of the disk-corona system.

\subsection{Calculation of the Spectra}

With determination of the disk and corona features, we are able to calculate the spectrum of the disk and corona. The contribution to the emergent spectra are composed of two components: (1) Multi black-body emission of the underlying thin disk which is partially scattered by the electrons in the hot corona (Compton emission) (2) Bremsstrahlung emission of the transition layer between the disk and corona. We use Monte Carlo simulation to calculate the Comptom spectra from the hot corona. The method of the Monte Carlo simulation is based on Pozdniakov, Sobol' \& Sunyaev (1977). We assume the electrons in the corona have a Maxwellian distribution, and the Compton scattering is the main cooling process. Since the electron-scattering optical depth in the corona is less than one, we introduce the weight $w$ as described by Pozdniakov et al. (1977) in order to efficiently calculate the effects of multiple scattering. We first set $w_{0}=1$ for a given soft photon, then calculate the escape probability $P_{0}$ of passing through the slab. The quantity of $w_{0} P_{0}$ are the trans-

mitted portion and is recorded to calculate the penetrated spectrum or reprocessed photons 
according to the escape direction of the photon. The remaining weight $w_{1}=w_{0}\left(1-P_{0}\right)$ is the portion that undergoes at least one scattering. If we write the escape probability after the $n$-th scattering as $P_{n}$, the quantity $w_{n} P_{n}$ is the transmitted portion of photons after the $n$-th scattering, and is recorded as upward or downward transmitted spectrum. The remaining portion $w_{n}\left(1-P_{n}\right)$ undergoes the $(n+1)$-th scattering. This calculation is continued until the weight $w$ becomes sufficiently small. The whole process is simulated by the Monte Carlo method.

Because the temperature of the transition layer is obviously higher than the underlying cool disk, we expect the inverse Compton scattering of the photons from the transition layer in the hot corona is not important Compared with the soft photons from the underlying cool disk. So, we ignore the inverse Compton scattering of the bremsstrahlung photons from the transition layer in the hot corona. The bremsstrahlung spectral flux from the transition layer per unit area can be expressed as,

$$
\begin{aligned}
F_{\nu}^{\mathrm{brem}} & =\frac{1}{2} j_{\nu} Z_{\mathrm{cpl}} \\
& =\frac{1}{2} \times 6.8 \times 10^{-38} Z^{2} n_{e} n_{i} T_{\mathrm{cpl}}^{-1 / 2} \overline{g_{\mathrm{ff}}}\left(\nu, T_{\mathrm{cpl}}\right) e^{-h \nu / k T_{\mathrm{cpl}}} Z_{\mathrm{cpl}} \\
& =f \overline{g_{\mathrm{ff}}} e^{-h \nu / k T_{\mathrm{cpl}}} \mathrm{ergs} \mathrm{s}^{-1} \mathrm{~cm}^{-2} \mathrm{~Hz}^{-1}
\end{aligned}
$$

where $j_{\nu}$ refers to the volume emissivity coefficient which is, $j_{\nu}=6.8 \times 10^{-38} Z^{2} n_{e} n_{i} T_{\mathrm{cpl}}^{-1 / 2}$ $\overline{g_{\mathrm{ff}}}\left(\nu, T_{\mathrm{cpl}}\right) e^{-h \nu / k T_{\mathrm{cpl}}}$, and $f=3.4 \times 10^{-38} Z^{2} n_{e} n_{i} T_{\mathrm{cpl}}^{-1 / 2} Z_{\mathrm{cpl}} . \mathrm{Z}_{\mathrm{cpl}}$ is the thickness of the transition layer, and $T_{\mathrm{cpl}}=1.98 \times 10^{9} \alpha^{-4 / 3} \dot{m}^{2 / 3} \mathrm{~K}$ is the electron temperature of the transition layer (Liu et al. 2007). In eq. (19), the coefficient $1 / 2$ means that only the upward photons from the transition layer can directly escape from the disk-corona system. The frequencyintegrated flux from the transition layer is,

$$
\begin{aligned}
F_{\text {brem }} & =\int_{0}^{\infty} F_{\nu}^{\mathrm{brem}} d \nu=f \int_{0}^{\infty} \overline{g_{\mathrm{ff}}}\left(\nu, T_{\mathrm{cpl}}\right) e^{-h \nu / k T_{\mathrm{cpl}}} d \nu \\
& =\frac{f k T_{\mathrm{cpl}}}{h} \int_{0}^{\infty} \bar{g}_{\mathrm{ff}}\left(\nu, T_{\mathrm{cpl}}\right) e^{-x} d x \quad \operatorname{ergs~s}^{-1} \mathrm{~cm}^{-2} .
\end{aligned}
$$

Solving eq. (20), we can get,

$$
f=\frac{F_{\mathrm{brem}} h}{k T_{\mathrm{cpl}}} \frac{1}{\int_{0}^{\infty} \bar{g}_{\mathrm{ff}}\left(\nu, T_{\mathrm{cpl}}\right) e^{-x} d x},
$$

the expression of $F_{\text {brem }}$ is given as (Liu et al. 2007),

$$
F_{\text {brem }}=\frac{1}{2} \times 6.391 \times 10^{24} \alpha^{-7 / 3} m^{-1} \dot{m}^{5 / 3} r^{-5 / 2} \mathrm{ergs} \mathrm{s}^{-1} \mathrm{~cm}^{-2},
$$


where the integral $\int_{0}^{\infty} \bar{g}_{\mathrm{ff}}\left(\nu, T_{\mathrm{cpl}}\right) e^{-x} d x$ is a frequency average of the velocity averaged Gaunt factor, which is in the range 1.1 to 1.5 . Choosing a value of 1.2 will give an accuracy to within about $12 \%$ (Rybicki \& Lightman 1979).

By combing eq.(19) and eq. (21), we get the local emergent bremsstrahlung spectra from the transition layer, which is given as,

$$
F_{\nu}^{\mathrm{brem}}=\frac{F_{\mathrm{brem}} h}{k T_{\mathrm{cpl}}} \frac{1}{\int_{0}^{\infty} \bar{g}_{\mathrm{ff}}\left(\nu, T_{\mathrm{cpl}}\right) e^{-x} d x} \overline{g_{\mathrm{ff}}}\left(\nu, T_{\mathrm{cpl}}\right) e^{-h \nu / k T_{\mathrm{cpl}}} \quad \operatorname{ergs~s}^{-1} \mathrm{~cm}^{-2} \mathrm{~Hz}^{-1}
$$

By integrating the local Compton spectra from the corona and the bremsstrahlung spectra from the transition layer along radial direction, we get the total emergent spectra of the inner disk-corona system.

\section{Numerical Results}

Given a black holes mass $m$, the mass accretion rate $\dot{m}$, the viscosity parameter $\alpha$ and the albedo $a$, the structure of the corona in radial direction is solved self-consistently as described in Section 2. Throughout the calculation, $m=10$ is adopted.

For viscosity parameter $\alpha=0.2$, and albedo $a=0.15$, it's found that the inner disk can survive for accretion rate in the range of $\dot{m} \sim 0.006-0.03$, which are consistent with the results of Liu et al (2011), where the modification to the cooling of the corona are not considered. This is because, at the upper limit of the accretion rate, the corona is dominated by the Compton cooling, the modification of conductive cooling to the corona can be neglected, and at the lower limit of the accretion rate, the corona is dominated by the Conductive cooling, the modification of Compton cooling to the corona can be neglected. The size of the accretion disk as functions of mass accretion rate is plotted in the left panel of Fig. 1 with red line. The size of the inner disk is defined by a critical radius, where neither the evaporation nor the condensation precess occurs. Outside the critical radius, the matter is evaporated to the corona from the disk (if the disk is not evaporated completely) and inside the critical radius, the corona matter condenses onto the disk. An increase of the mass accretion rate leads to more gases condensation onto the disk and the corresponding critical radius of the inner disk increases. The maximum temperature of the inner disk is presented in the right panel of Fig. 1 with red line. It's evident that the maximum temperature of the disk increases with the mass accretion rate. This is because, an increased value of the mass accretion rate results in both the increase of the condensation rate and the luminosity of the hot corona, the maximum temperature of the inner disk heated up by accreting condensed gas and the irradiation of the hot corona integrated throughout the inner disk increases. The 
ratio of luminosity dissipated in the corona to the luminosity dissipated in the disk, $L_{\mathrm{c}} / L_{\mathrm{d}}$, as functions of mass accretion rates $\dot{m}$ is plotted in Fig. 2 with red line. With increase of the accretion rate $\dot{m}$, the relatively quick increase of the emission from the accretion disk to the emission from the corona leads to a decreased value of $L_{\mathrm{c}} / L_{\mathrm{d}}$. The electron temperature and the optical depth for the Compton scattering of the hot electron in the vertical direction as functions of radius are plotted in Fig. 3 with red line. The solid line is for $\dot{m}=0.03$ (dotted line $\dot{m}=0.02$; dashed line: $\dot{m}=0.01$ ). We can see the electron temperatures are in the range of $\sim 1-5 \times 10^{9} \mathrm{~K}$ for different accretion rate $0.01 \leq \dot{m} \leq 0.03$. It's clear that the temperature of the corona decreases with increase of the mass accretion rate for $a=0.15$. This is because, with increase of the mass accretion rate, the Compton cooling of the soft photons from the disk to the corona becomes more efficient, the temperature of the corona decreases. To clearly show the mass distribution of the inner disk-corona system in radial direction, we plot the mass accretion rate in the accretion disk and the mass accretion rate in the corona as functions of radius in Fig. 4. It can be seen that, with increase of the mass accretion rate, more gases in the corona recondense back to the disk, the accretion rate in the disk increases.

The emergent spectra of inner disk and corona with mass accretion rate are plotted in Fig. 5. The X-ray spectrum in the range of $2-10 \mathrm{keV}$ is roughly characterized by a power law with a photon index $\Gamma=1.63$ at $\dot{m}=0.01$ (solid green line). With increase of the mass accretion rate, the hard X-ray photon index is $\Gamma=1.75$ at $\dot{m}=0.02$ (solid blue line) and $\Gamma=1.92$ at $\dot{m}=0.03$ (solid red line). It's obviously that the X-ray spectra become softer with increase of the mass accretion rate. This can be understood as, the hard X-rays are dominated by Bremsstrahlung at low accretion rate, whereas by Compton radiation at high accretion rate. In Fig. 5, the dashed line is the contribution of the Compton scattering of the soft photons from the underlying cool disk by the electrons in the hot corona (Compton radiation), and the dotted line is the contribution from the bremsstrahlung of the transition layer. At lower accretion rate $\dot{m}=0.01$, the cooling of the corona is dominated by the conductive cooling, fewer soft photons from the underling thin disk are scattered to the hard X-ray band, so the Compton emission to the hard X-ray band is weak, the hard X-ray emission is dominated by the bremsstrahlung of the transition layer. With increase of the mass accretion rate to $\dot{m}=0.02$, the Compton emission of the corona to the X-ray band becomes dominant compared to the bremsstrahlung of the transition layer, as shown by the blue dashed line and dotted line in Fig. 5. With further increase of accretion rate, the Compton $y$-parameter decreases, which results in a large photon index and small luminosity ratio between the corona and disk, as shown by the red solid line in Fig. 5 and Fig. 2 ,

In order to study the effect of albedo $a$ on the spectra, we plot the emergent spectra of the inner disk and corona with albedo in Fig. 6. The size and the maximum effective 
temperature of the inner accretion disk with a can also be seen in Fig 1. The electron temperature and the optical depth for the Compton scattering of the hot electron in the vertical direction as functions of radius are plotted in Fig. 3. The red line, blue line and green line are for albedo $a=0.15, a=0.6$ and $a=1$ (without irradiation) respectively. For viscosity parameter $\alpha=0.2$ and mass accretion rate $\dot{m}=0.02$, we plot the emergent spectra for different albedo $a$ in Fig. 6. For albedo $a=0.15$, the hard X-ray photon index $\Gamma=1.75$. With increase the value of albedo, the hard $\mathrm{X}$-ray photon index are $\Gamma=1.63,1.53$ for $a=0.6,1$ respectively. It can be seen clearly, X-ray spectrum becomes harder with increase of albedo. This is because for larger albedo, fewer X-ray photons of the irradiation from the corona to the disk are absorbed and reprocessed as thermal seed photons to be scattered in the corona, the temperature of the electron in the corona is higher, a harder $\mathrm{X}$-ray spectrum is predicted. We plot the emergent spectra with albedo $a$ for different mass accretion rate in Fig. 7. We can see, for both $\dot{m}=0.01$ and $\dot{m}=0.03$, the hard X-ray spectra become harder with increase of $a$. From Fig. 6, it's also very clear that the positive dependence of the hard X-ray spectrum index on the mass accretion rate holds for different albedo $a$.

From theory, the value of albedo $a$ is uncertain, and has been investigated by several authors, e.g. George \& Fabian (1991), White et al. (1988). The value of albedo $a$, which depends on how the incident photons from the corona interact with the underlying accretion disk, is defined as the energy in the reflective spectrum divided by the energy in the incident spectrum. The value of albedo is energy-dependent. White et al. (1988) study the Compton scattering of the incident photons by the electrons in the disk. In their calculations, X-rays is assumed to incident upon a semi-infinite, plane-parallel, zero temperature, purely scattering medium. They show that the monochromatic albedo $a\left(x_{0}\right)$ changes from $95 \%$ to $1 \%$ for the energy $x_{0}$ in the range of $\sim 10^{-3}$ to $\sim 30$ ( $x_{0}$ is the energy of the incident photons scaled with rest energy of electron). If the mean energy of the photons are around $50 \mathrm{keV}$, $\sim 40 \%$ of the irradiation flux is absorbed by the disk through the Compton scattering of the electron in the disk (White et al. 1988). The K-shell absorption of the metals is negligible for the incident photons at $\sim 50 \mathrm{keV}$. However, because the K-shell absorption of the metals is proportional to $\left(m_{e} c^{2} / h \nu\right)^{7 / 2}$, at lower energy, the K-shell absorption becomes important. For the distribution of $N_{\nu} \propto \nu^{-1.6}, \sim 30 \%$ of the incident energy is absorbed (Taam et al. 2008). Thus, $\sim 70 \%$ of the total irradiation flux is absorbed by the Compton scattering and K-shell absorption, so the value of albedo $a$ is around 0.3. However, it's highly uncertain for the calculation the value of albedo, e.g. the ionization state of the surface of the accretion disk. In this paper, we only take albedo as a parameter to fit the observations.

To test the effect of the viscosity parameters $\alpha$ to the shape of the spectra, we plot the emergent spectra of the inner disk and corona with viscosity parameter $\alpha$ in Fig. 8 , In our 
calculation, albedo $a=0.15$ and mass accretion rate $\dot{m}=0.03$ are adopted respectively. For $\alpha=0.2$, the hard X-ray photons is $\Gamma=1.92$. With increase of $\alpha$, the hard X-ray photon index are $\Gamma=1.67,1.57$ for $\alpha=0.25,0.3$ respectively. It's found that the luminosity of the disk-corona system decreases systematically with the value of $\alpha$, meanwhile, the X-ray spectra in the range of $2-10 \mathrm{keV}$ becomes harder with the value of $\alpha$. This is because, for fixed mass accretion rate, the surface density in the corona decreases with increase of $\alpha$. With a lower density, the heat flux to the transition layer decreases, meanwhile the bremsstrahlung cooling rate is decreased even more. The energy balance between heating and cooling results in a decreased heating rate associated with a lower enthalpy flux. The net effect results in a decreased condensation rate. The energy conversion efficiency of the inner disk + corona/ADAF can be expressed as $\eta / 0.1=\left(L / L_{\text {Edd }}\right) /\left(\dot{M} / \dot{M}_{\text {Edd }}\right)$. The efficiency of the thin disk is 0.1 , however, for the hot corona/ADAF, due to the strong advection effect, the efficiency is much less than 0.1 . With increase of $\alpha$, due to the decrease of the condensation rate, less matters are accreted in the form of thin disk, the luminosity of the inner disk + corona/ADAF system decreases. Meanwhile, due to relative lack photons from the underlying thin disk to be scattered in the corona, the temperature of the corona is higher, so a harder X-ray spectrum is predicted.

To show the effect of the different parameters (accretion rate $\dot{m}$, viscosity parameter $\alpha$ and albedo $a$ ) to the condensation features and the corresponding spectral features of the inner disk and corona clearly, we list the numerical results for different parameters in Table 1.

\section{Comparison To Observations}

\subsection{GX 339-4}

The distance to GX 339-4 is dynamically constrained at $6.7<d<9.3 \mathrm{kpc}$ (Zdziarski et al. 2004), which is consistent with the lower limit of $d \gtrsim 6 \mathrm{kpc}$ for kinematical distance based on analysis of high-resolution spectra of the Na D line (Hynes et al. 2004). The mass function of GX 339-4 is constrained to $\sim 6 M_{\odot}$ (Hynes et al. 2003; Muñoz-Darias et al. 2008). In this paper, a likely distance of $d=8 \mathrm{kpc}$ and mass $m=5.8$ are adopted to fit the recent observations (Tomsick et al. 2008). A thermal component with an inner disk temperature of $0.165 \mathrm{keV}$ at a lower luminosity $L \approx 0.008 L_{\mathrm{Edd}}(1-100 \mathrm{keV})$ are detected by the observations of Swift and RXTE during 2007 June 10-14. The most constraints of the disk come from the reflection component, which requires that the disk can extend to $r<5$ (Tomsick et al. 2008). 
With $m=5.8$, the X-ray luminosity $L=0.008 L_{\text {Edd }}$ and effective temperature of the disk $T_{\text {eff,max }}=0.165$ from observations. We assume viscosity parameter $\alpha=0.3$, and find that the observed X-ray luminosity and the effective temperature are matched by adopting $\dot{m}=0.0456$ and albedo $a=0.782$ respectively. The fitting results are listed in Table 2 . We calculate the emergent spectra of the inner disk-corona system with the fitting parameters, and plot the spectrum in Fig. 9 (the solid line). It's found that the hard X-ray spectrum in the range of $1-100 \mathrm{keV}$ is roughly characterized by a power law with a photon index $\Gamma_{\text {mod }}=1.50$ from our model, which is roughly close to the observed one $\Gamma=1.63_{-0.03}^{+0.04}$ (Tomsick et al. 2008).

With increase of the viscosity parameter to $\alpha=0.4$, we find the observed X-ray luminosity and the maximum effective temperature of the disk can be matched by taking accretion rate $\dot{m}=0.0773$ and albedo $a=0.617$ respectively. In this case, the condensation rate is $\dot{m}_{\text {cnd }}=5.98 \times 10^{-4}$ which is smaller than $\dot{m}_{\text {cnd }}=1.4 \times 10^{-3}$ for the case $\alpha=0.3$. So from eqs. (16) and (17), a smaller value of albedo $a$ is needed to match the observed effective temperature. We plot the corresponding emergent spectrum in Fig 9 (the dotted line). The hard X-ray spectrum from $1-100 \mathrm{keV}$ is also roughly characterized by a power law with a photon index $\Gamma_{\text {mod }}=1.4$ from our model. The detailed fitting results are shown in Table 2 .

From the calculation above, it's found that, the hard X-ray photon index calculated by taking $\alpha=0.3$ can match the observed one better than that of taking $\alpha=0.4$. Here, we want to show that the observed X-ray luminosity and the maximum effective temperature of the disk can only be fitted in a very narrow range for the value of $\alpha$. For instance, for $\alpha=0.2$, we can't fit the X-ray luminosity and the maximum effective temperature simultaneously, i.e., by taking $\dot{m}=0.0251$, the observed X-ray luminosity can be matched, however, even by taking the limited value of albedo $a=1$, the theoretical value of $T_{\text {eff,max }}$ is $0.1825 \mathrm{keV}$ which is bigger than the observed value $0.165 \mathrm{keV}$.

\section{2. $\mathrm{Cyg} \mathrm{X}-1$}

Cyg X-1 is a well-known black hole X-ray binaries and has been well studied by several authors. The distance to Cyg X-1 is very early estimated at 2 kpc (Murdin \& Webster 1971; Reis et al. 2010), and confirmed by Massey et al. (1995) where a distance of $2.1 \pm 0.1 \mathrm{kpc}$ is found. The mass of Cyg X-1 is found in the range of $7-15 M_{\odot}$ (Shaposhnikov \& Titarchuk 2009). In this paper, a distance $d=2 \mathrm{kpc}$ and $m=10$ are adopted (Reis et al. 2010). We compare our result with the recent observations of Cyg X-1 (Reis et al. 2010). They find that an X-ray luminosity $L_{\mathrm{x}} / L_{\mathrm{Edd}}=5 \times 10^{-3}(\mathrm{~m} / 10) \times(d / 2 \mathrm{kpc})^{2}$ in the range of $0.5-10 \mathrm{keV}$ and a hard power-law photon index $\Gamma=1.71 \pm 0.01(2-10 \mathrm{keV})$. Extrapolating the luminosity 
to $100 \mathrm{keV}$, we get a X-ray luminosity $L_{\mathrm{x}} / L_{\mathrm{Edd}}=1.47 \times 10^{-2}(\mathrm{~m} / 10) \times(d / 2 \mathrm{kpc})^{2}$. By fitting the spectra with Diskbb+Power law model, they reveal a cool disk component with a temperature $k T \approx 0.194 \mathrm{keV}$, meanwhile it's found that the inner boundary of the accretion disk is very close to ISCO, i.e., $r_{\text {in }}=5.7_{3.0}^{+7.0}$ (Reis et al. 2010).

With $m=10$, the X-ray luminosity $L_{\mathrm{x}}=1.47 \times 10^{-2} L_{\mathrm{Edd}}$ and effective temperature of the disk $k T \approx 0.194 \mathrm{keV}$ derived from observations, and assuming viscosity parameter $\alpha=0.3$, it's found that the observed X-ray luminosity and effective temperature are matched by taking $\dot{m}=0.044$ and albedo $a=0.398$ respectively. We plot the emergent spectrum of the inner disk and corona with the fitting parameters in Fig. 10 (the solid line). It's found that the hard X-ray spectra in the range of $2-10 \mathrm{keV}$ is characterized by a power law with a photos index $\Gamma_{\text {mod }}=1.60$ from our model. The fitting results for bigger viscosity parameter $\alpha=0.4$ are also listed in Table. 2 .

\section{Conclusion}

We study the formation of a cool, optically thick, geometrically thin disk in the most inner region of black hole X-ray binaries in the low/hard spectral state within the context of disk accretion fed by condensation of hot corona. By taking into account the cooling process associated with both Compton scattering and vertical conduction in a corona, and the irradiation of the hot corona to the disk, we obtain a self-consistent solution of the inner disk and corona. We calculate the emergent spectra of the inner disk and corona for different accretion rate, and examine the effect of different parameters on the spectra (e.g. viscosity parameters $\alpha$ and albedo $a$ ). Our model is used to explain the spectral features of BHXRBs GX 339-4 and Cyg X-1 in the low/hard spectral state, in which the thin disk resides in the region very closed to the ISCO. Our results are roughly in agreement with the observations characterized by a weak disk component with temperature of $\sim 0.2 \mathrm{keV}$ and a hard power-law X-ray spectra with $\Gamma \sim 1.5-2.1$ for the sources in the low/hard spectral state.

We thank Prof. Ronald E. Taam for his very useful suggestions and comments. We thank the referee for his/her expert comments and suggestions to our paper. This work is supported by the the National Natural Science Foundation of China (grants 11033007, 11173029) and by the National Basic Research Program of China-973 Program 2009CB824800. 


\section{REFERENCES}

Abramowicz, M.A., Chen, X. Kato, S. et al. 1995, ApJ, 438, L37

Done, C., Gierliński, Marek, \& Kubota, Aya, 2007, A\&A Rev., 15, 1

Esin, A.A, McClintock J.E., \& Narayan, R. 1997, ApJ, 489, 865

Frank, J., King, A., \& Raine, D. 2002, Accretion Power in Astrophysics, Cambridge Univ. Press

George, I. M., \& Fabian, A. C., 1991, MNRAS, 249, 352

Gilfanov, M., 2009, to appear in "The Jet Paradigm - From Microquasars to Quasars", ed. T. Belloni, Lect. Notes Phys. 794

Hynes, R. I., Steeghs, D., Casares, J., Charles, P. A., \& O’Brien, K., 2003, ApJ, 583, L95

Hynes, R. I., Steeghs, D., Casares, J., Charles, P. A., \& O’Brien, K., 2004, ApJ, 609, 317

Kato, S. Fukue, J. \& Mineshige, S. 2008, "Black-Hole Accretion Disks", Kyoto University Press (Kyoto)

Kalemci, E., et al., 2007, The Astronomer's Telegram, 1074

Kawabata, Ryoji, \& Mineshige, Shin, 2010, PASJ, 62, 621

King, A. R., Pringle, J. E., \& Livio, M. 2007, MNRAS, 376, 1740

Liu, B. F., Yuan, W., Meyer, F., Meyer-Hofmeister, E., \& Xie, G. Z. 1999, ApJ, 527, L17

Liu, B. F., Mineshige, S., Meyer, F., Meyer-Hofmeister, E., \& Kawaguchi, T. ApJ, 2002, 575,117

Liu, B. F., Meyer, F., \& Meyer-Hofmeister, E. 2006, A\&A, 454, L9

Liu, B. F., Taam, R. E., Meyer, F., \& Meyer-Hofmeister, E. 2007, ApJ, 671, 695

Liu, B. F., Done, C., \& Taam, Ronald E., 2011, ApJ, 726, 10L

Massey, Philip, Johnson, Kelsey E., \& Degioia-Eastwood, Kathleen, 1995, ApJ, 454, 151

Mitsuda, K., Inoue, H., Koyama, K., Makishima, K., Matsuoka, M., Ogawara, Y., Suzuki, K., Tanaka, Y., Shibazaki, N., \& Hirano, T. 1984, PASJ, 36, 741

Meyer, F., Liu, B.F., \& Meyer-Hofmeister, E. 2000a, A\&A, 361, 175 
Meyer, F., Liu, B.F., \& Meyer-Hofmeister, E. 2000b, 354, L67

Meyer, F., Liu, B. F., \& Meyer-Hofmeister, E. 2007, A\&A, 463, 1

Muñoz-Darias, T., Casares, J., \& Martínez-Pais, I. G. 2008, MNRAS, 385, 2205

Murdin P., \& Webster L. B., 1971, Nat, 233, 110

Narayan, R.,\& Yi, I. 1994, ApJ, 428, L13

Narayan, R.,\& Yi, I. 1995a, ApJ, 444, 231

Narayan, R., \& Yi, I. 1995b, ApJ, 452, 710

Narayan, R. 2005, Ap\&SS, 300, 177

Narayan, R., \& McClintock, J. E. 2008, New Astron. Rev., 51, 733

Pozdniakov, L. A., Sobol, I. M., \& Siuniaev, R. A. 1977, Soviet Astronomy, 21, 708

Pringle, J. E., \& Rees, M. J. 1972, A\&A, 21, 1

Qiao, Erlin, \& Liu, B. F. 2009, PASJ, 61, 403

Qiao, Erlin, \& Liu, B. F. 2010, PASJ, 62, 661

Rees, M. J., Belgman, M. C., Blandford, R. D., \& Phinney, E. S. 1982, Nature, 295, 17

Reis, R. C., Fabian, A. C., \& Miller, J. M., 2010, MNRAS, 402, 836

Remillard, R. A. \& McClintock, J. E. 2006. Annu. Rev. Astron. Astrophys.

Shakura, N.I., \& Sunyaev, R. A. 1973, A\&A, 24, 337

Shaposhnikov, Nickolai, \& Titarchuk, Lev, 2009, ApJ, 699, 453

Taam, R. E., Liu, B.F., Meyer, F., \& Meyer-Hofmeister, E. 2008, ApJ, 688, 527

Thorne, K. S., 1974, ApJ, 191, 507

Tomsick, J. A. et al. 2008, ApJ, 680, 593

White, Timothy R., Lightman, Alan P., \& Zdziarski, Andrzej A., 1988, ApJ, 331, 939

Zdziarski, A. et al. 2004, MNRAS, 351, 791 

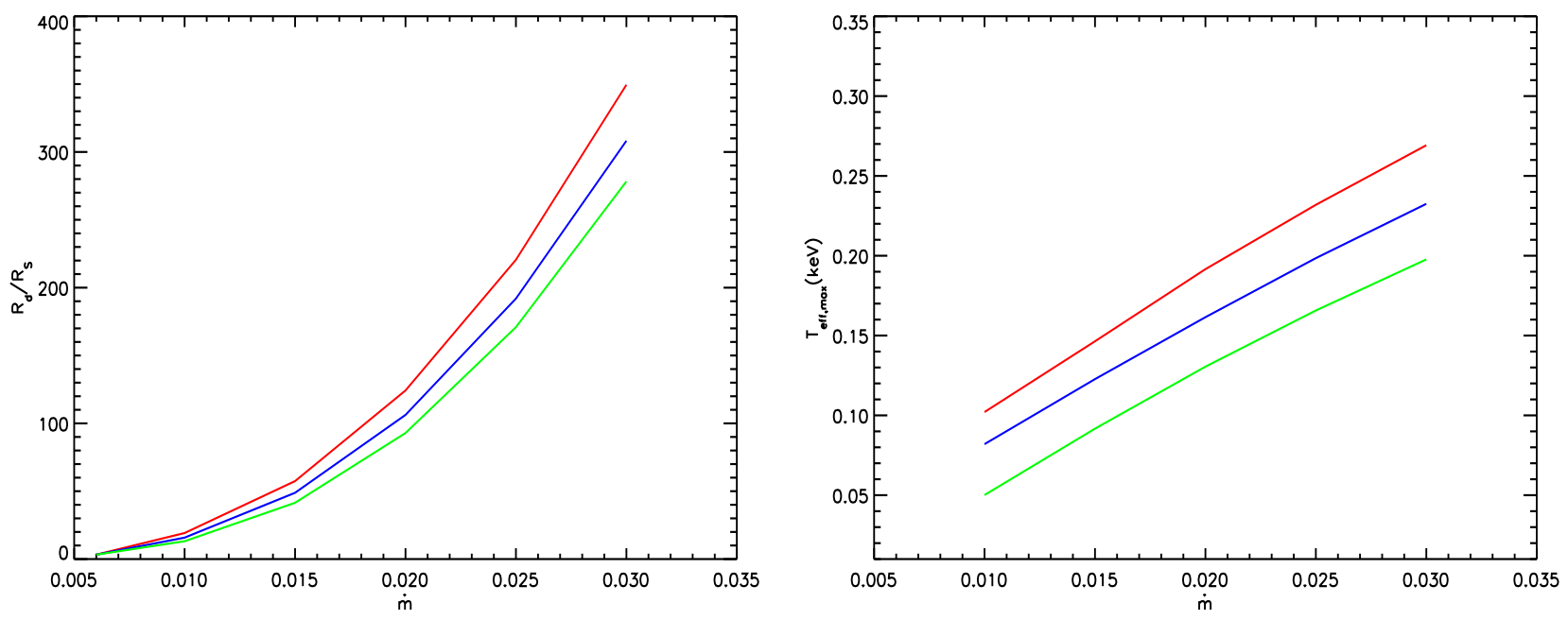

Fig. 1. - The left panel: The size of the inner accretion disk as functions of mass accretion rate $\dot{m}$. In our calculation, $\alpha=0.2$ is adopted. The red line is for albedo $a=0.15$ (blue line: $a=0.6$; green line: $a=1$ ). The right panel: The maximum temperature of the disk as functions of mass accretion rate $\dot{m}$.

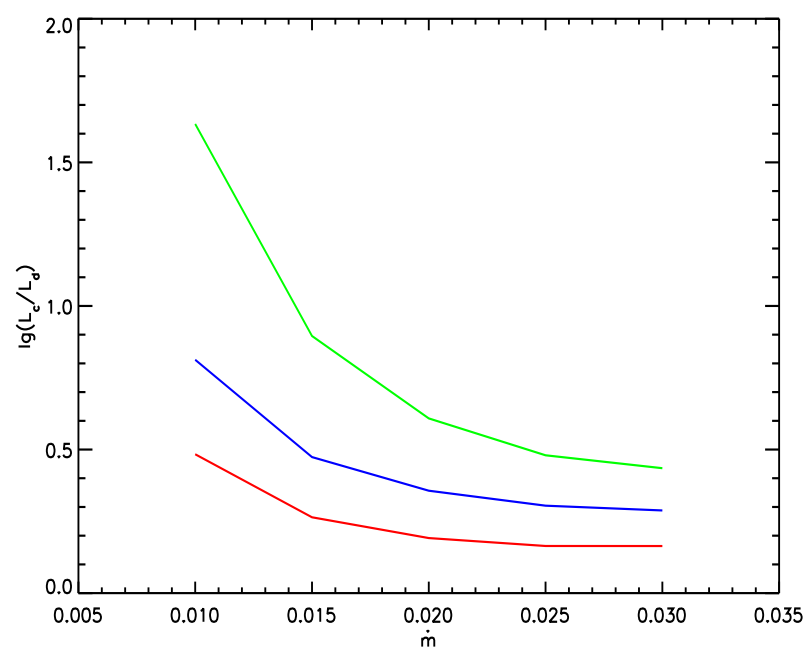

Fig. 2.- The ratio of $L_{\mathrm{c}} / L_{\mathrm{d}}$ as functions of mass accretion rate $\dot{m}$. In our calculation, $\alpha=0.2$ is adopted. The red line is for albedo $a=0.15$ (blue line: $a=0.6$; green line: $a=1)$. 

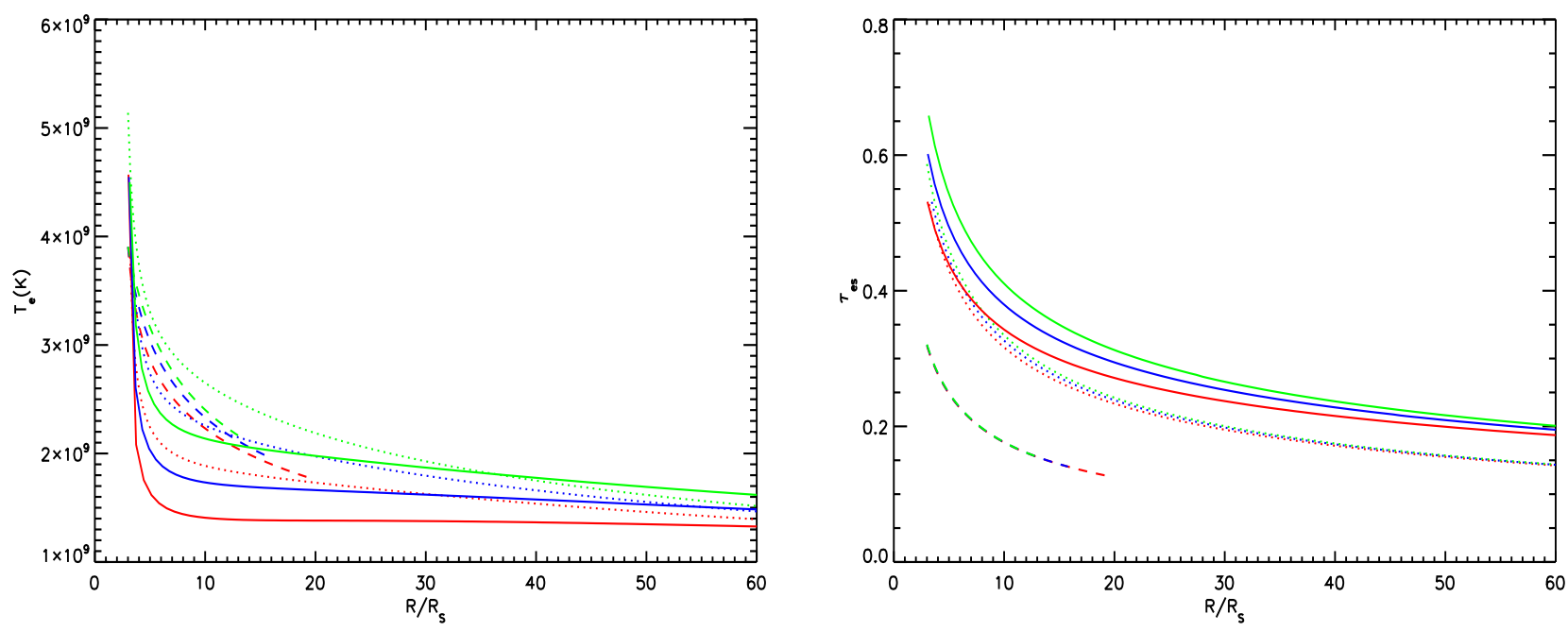

Fig. 3. - The left panel: the electron temperature of the corona as a function of radius. In our calculation, $\alpha=0.2$ is adopted. The red lines represent the results calculated for albedo $a=0.15$ (blue lines: $a=0.6$; green line: $a=1$ ). The different line types represent different accretion rates (dashed line: $\dot{m}=0.01$; dotted line $\dot{m}=0.02$; solid line: $\dot{m}=0.03$ ). The right panel: the Thomson optical depth for the Compton scattering of the hot corona in the vertical direction as a function of radius.

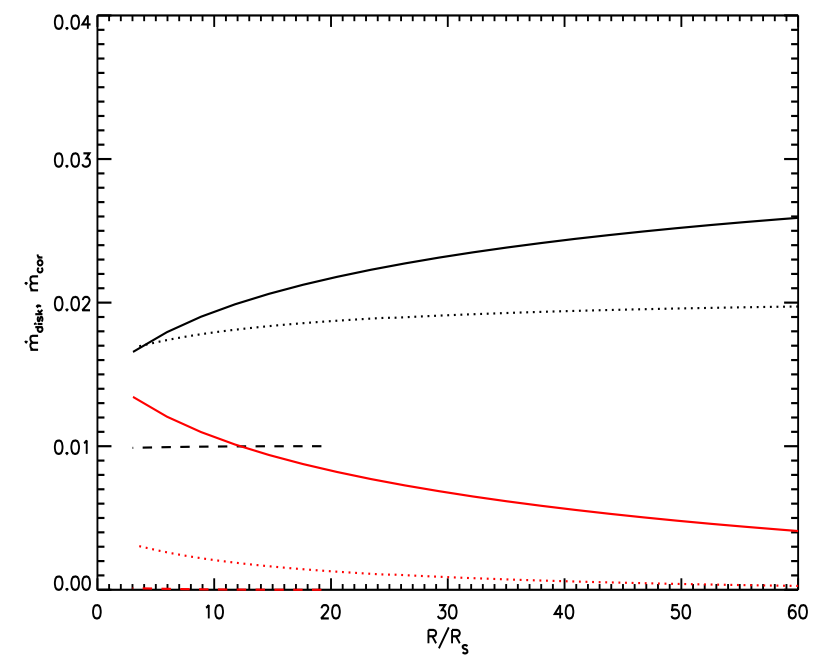

Fig. 4. - The mass accretion rate in the accretion disk and the mass accretion rate in the corona as functions of radius. The red line and black line represent the mass accretion rate in the disk and the mass accretion rate in the corona respectively. The solid line, dotted line and dashed line are for $\dot{m}=0.03, \dot{m}=0.02$ and $\dot{m}=0.01$ respectively. In our calculation, $\alpha=0.2$ and albedo $a=0.15$ are adopted. 


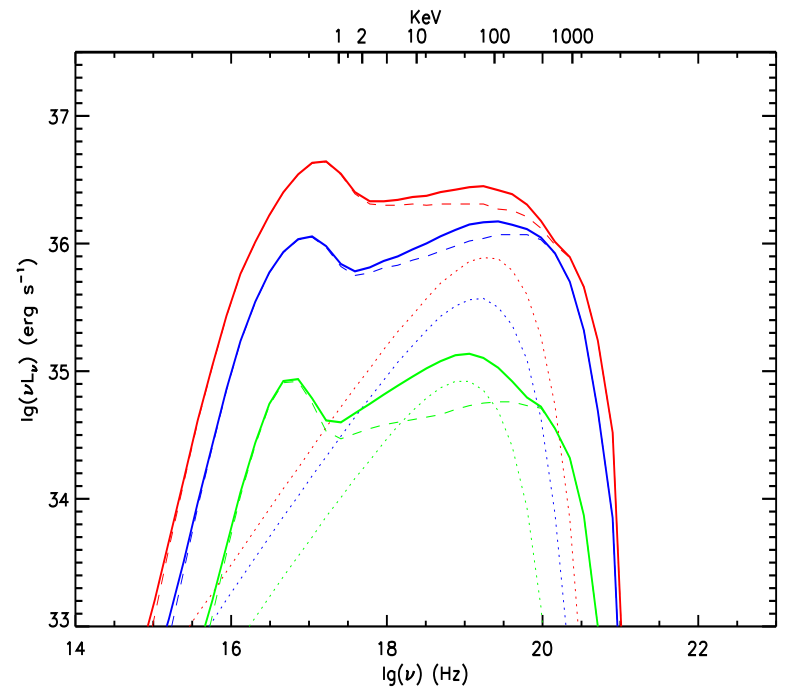

Fig. 5.- The emergent spectra of inner disk and corona for different mass accretion rate. In our calculation, $\alpha=0.2$ and albedo $a=0.15$ are adopted. The red line, blue line and green line are the spectra for $\dot{m}=0.03, \dot{m}=0.02$ and $\dot{m}=0.01$ respectively. The solid line is the total spectrum, the dashed line is the contribution from the Comptonization of the soft photons of the thin disk by the electrons in the hot corona, the dotted line is the contribution of the bremsstrahlung from the transition layer. 


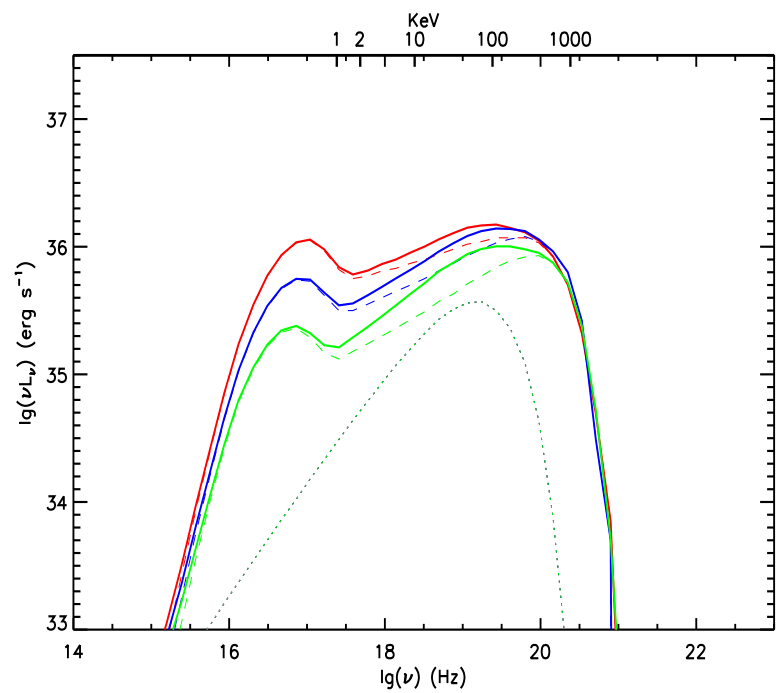

Fig. 6. - The emergent spectra of inner disk and corona for different albedo $a$. In our calculation, $\dot{m}=0.02$ and $\alpha=0.2$ are adopted respectively. The solid red line is the total spectrum for $a=0.15$, the red dashed line is the contribution from the Comptonization of the soft photons of the thin disk by the hot corona, the red dotted line is the contribution of the bremsstrahlung from the transition layer. The solid blue line and green line are for $a=0.6$ and $a=1$ respectively.

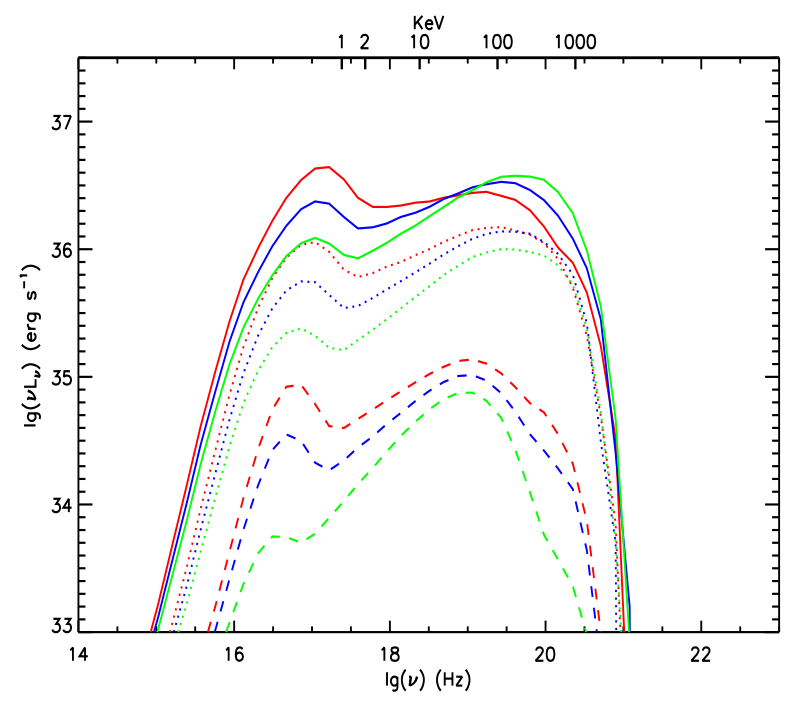

Fig. 7.- The emergent spectra of inner disk and corona for different accretion rate and albedo. In our calculation, $\alpha=0.2$ is adopted. The solid line, dotted line and dashed line are for $\dot{m}=0.03, \dot{m}=0.02$, and $\dot{m}=0.01$ respectively. The red line, blue line and green line are for albedo $a=0.15, a=0.6$ and $a=1$ respectively. 


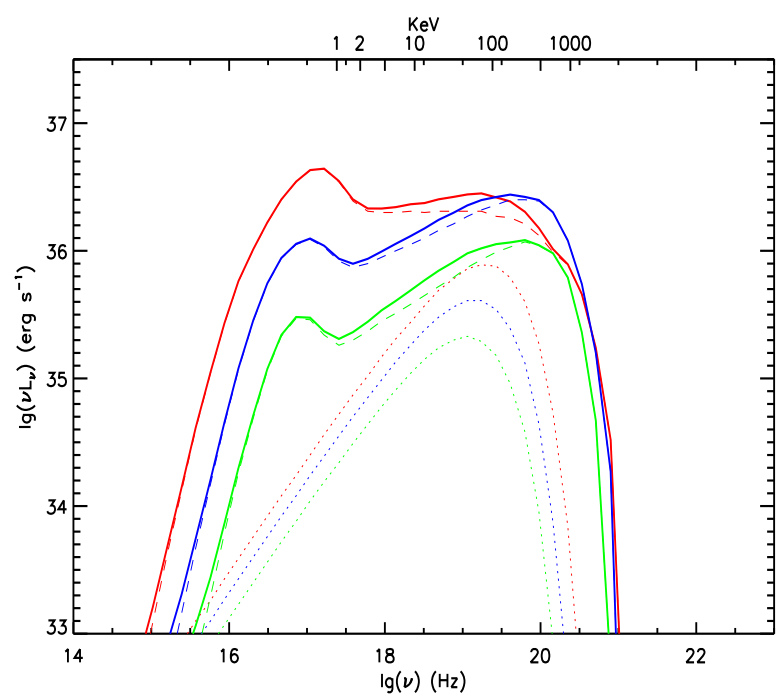

Fig. 8.- The emergent spectra of inner disk and corona for different viscosity parameter $\alpha$. In our calculation, $\dot{m}=0.03$ and $a=0.15$ are adopted. The red solid line is the total spectrum for $\alpha=0.2$, the red dashed line is the contribution from the Comptonization of the soft photons of the thin disk by the hot corona, the red dotted line is the contribution of the bremsstrahlung from the transition layer. The solid blue line and green line are for $\alpha=0.25$ and $\alpha=0.3$ respectively.

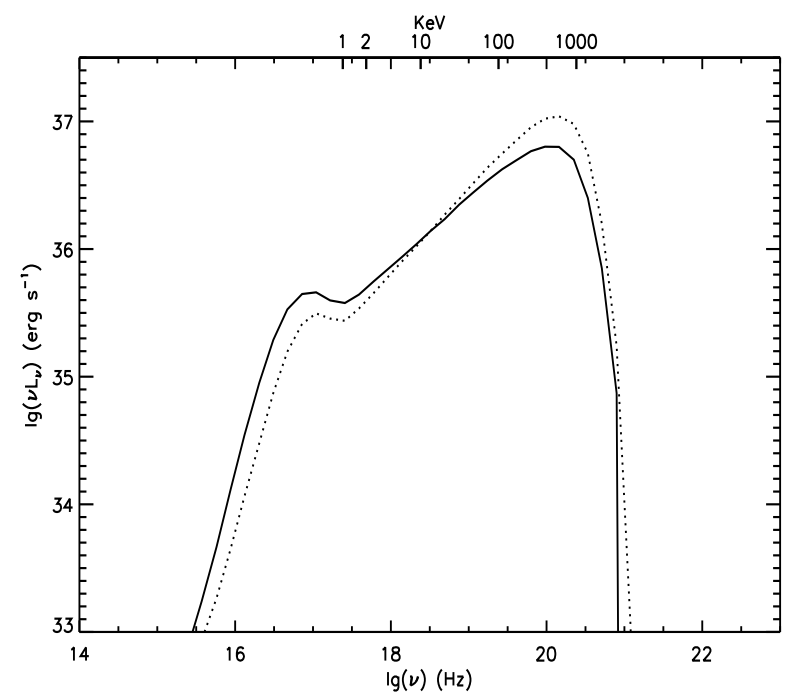

Fig. 9. - The emergent spectra of GX 339-4 calculated by different fitting parameters of our model. The solid line is the emergent spectrum for $m=5.8, \alpha=0.3, \dot{m}=0.0456$ and $a=0.782$; The dotted line is the emergent spectrum for $m=5.8, \alpha=0.4, \dot{m}=0.0773$ and $a=0.617$. 
Table 1: Condensation and spectral features of the inner disk and corona around the black hole of $10 M_{\odot}$

\begin{tabular}{ccc|cccccc}
\hline \hline & & & & & & & & \\
$\alpha$ & $a$ & $\dot{m}$ & $r_{\mathrm{d}}$ & $\dot{m}_{\text {cnd }}$ & $L_{\text {x (cor) }} / L_{\text {Edd }}$ & $L_{\mathrm{c}} / L_{\mathrm{d}}$ & $T_{\text {eff,max }}(\mathrm{keV})$ & $\Gamma_{\text {mod }}$ \\
\hline & & & & & & & & \\
0.2 & 0.15 & 0.01 & 19.2 & $9.66 \times 10^{-5}$ & $1.02 \times 10^{-3}$ & 3.04 & 0.10 & 1.63 \\
0.2 & 0.15 & 0.02 & 124.2 & $2.98 \times 10^{-3}$ & $9.18 \times 10^{-3}$ & 1.56 & 0.19 & 1.75 \\
0.2 & 0.15 & 0.03 & 350.0 & $1.29 \times 10^{-2}$ & $3.32 \times 10^{-2}$ & 1.45 & 0.27 & 1.92 \\
\hline & & & & & & & & \\
0.2 & 0.6 & 0.01 & 15.8 & $5.92 \times 10^{-5}$ & $8.22 \times 10^{-4}$ & 6.50 & 0.08 & 1.52 \\
0.2 & 1 & 0.01 & 13.1 & $3.60 \times 10^{-5}$ & $6.79 \times 10^{-4}$ & 43.0 & 0.05 & 1.40 \\
0.2 & 0.6 & 0.02 & 106.2 & $2.25 \times 10^{-3}$ & $6.71 \times 10^{-3}$ & 2.27 & 0.16 & 1.63 \\
0.2 & 1 & 0.02 & 92.9 & $1.66 \times 10^{-3}$ & $5.05 \times 10^{-3}$ & 4.06 & 0.13 & 1.53 \\
0.2 & 0.6 & 0.03 & 308.2 & $1.07 \times 10^{-2}$ & $2.48 \times 10^{-2}$ & 1.93 & 0.23 & 1.77 \\
0.2 & 1 & 0.03 & 278.3 & $8.72 \times 10^{-3}$ & $1.83 \times 10^{-2}$ & 2.72 & 0.20 & 1.62 \\
\hline & & & & & & & & \\
0.2 & 0.15 & 0.03 & 350.0 & $1.29 \times 10^{-2}$ & $3.32 \times 10^{-2}$ & 1.45 & 0.27 & 1.92 \\
0.25 & 0.15 & 0.03 & 83.0 & $3.19 \times 10^{-3}$ & $1.35 \times 10^{-2}$ & 1.79 & 0.21 & 1.67 \\
0.3 & 0.15 & 0.03 & 22.2 & $4.66 \times 10^{-4}$ & $4.25 \times 10^{-3}$ & 2.80 & 0.15 & 1.57 \\
\hline
\end{tabular}

Note.- With black hole mass $m=10$, viscosity parameters $\alpha$, albedo $a$, and the mass accretion rate $\dot{m}$, the size of the inner disk $r_{\mathrm{d}}$, condensation rate $\dot{m}_{\text {cnd }}$ integrated from the condensation radius to $3 R_{\mathrm{S}}$, the luminosity dissipated in the corona $L_{\mathrm{x}(\mathrm{cor})} / L_{\mathrm{Edd}}$, the ratio of luminosity dissipated in the corona to the luminosity dissipated in the disk $L_{\mathrm{c}} / L_{\mathrm{d}}$, the maximum temperature of the inner disk $T_{\text {eff,max }}$ and the hard X-ray photon index $\Gamma_{\bmod }$ in the range of 2-10 $\mathrm{keV}$ are listed. 
Table 2: Fitting Results for GX 339-4 and Cyg X-1

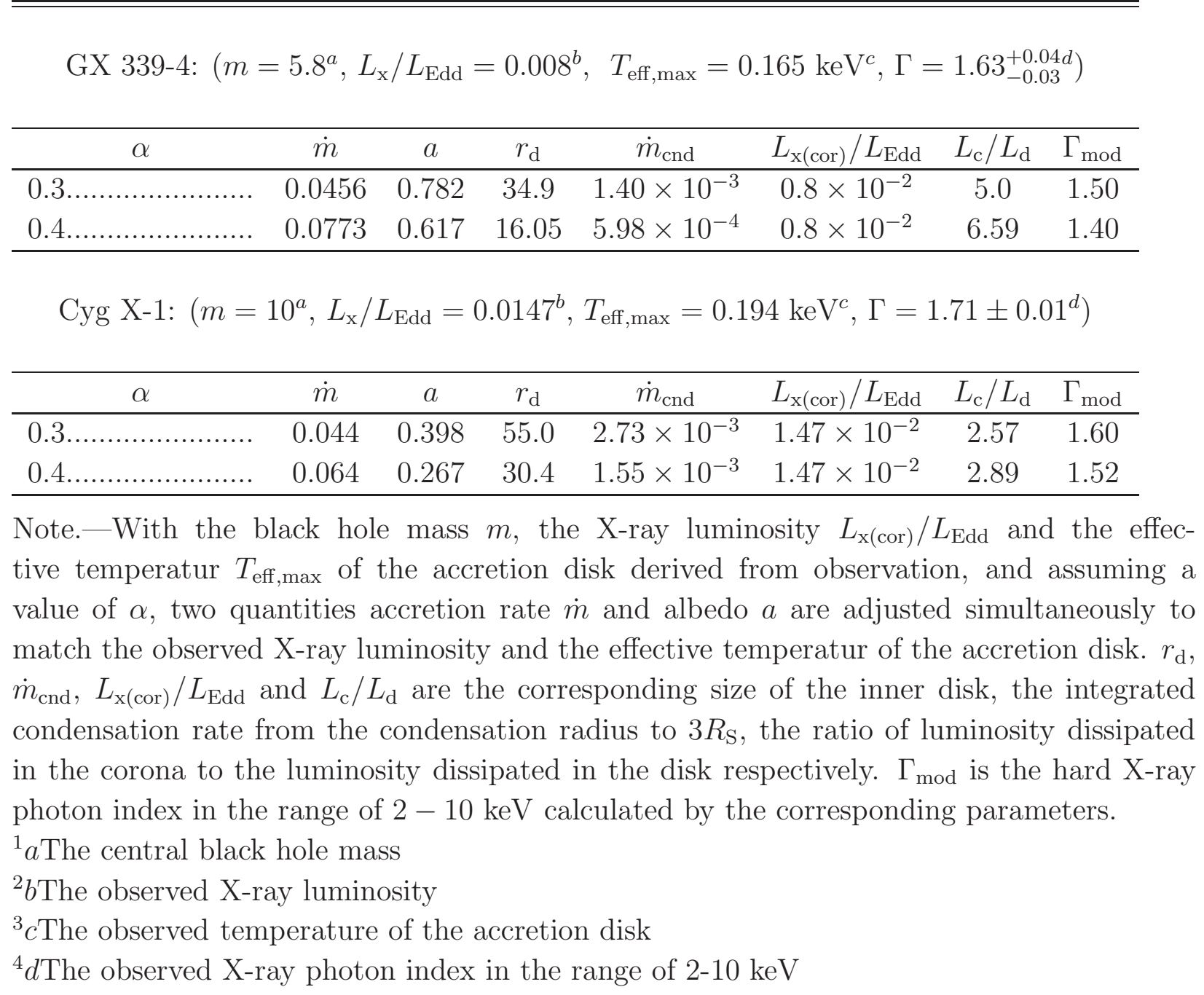




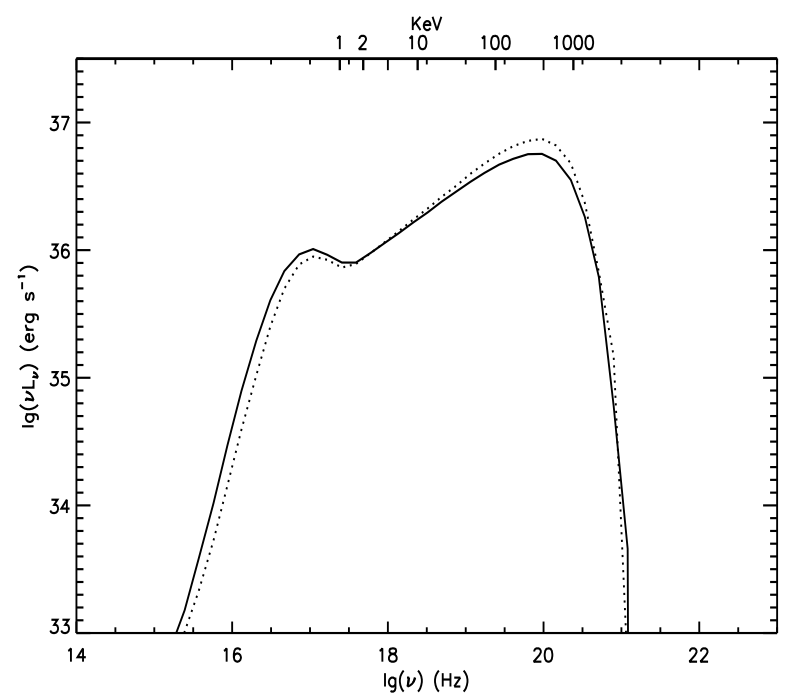

Fig. 10. - The emergent spectra of Cyg X-1 calculated by different fitting parameters of our model. The solid line is the emergent spectrum for $m=10, \alpha=0.3, \dot{m}=0.044$ and $a=0.398$; The dotted line is the emergent spectrum for $m=10, \alpha=0.4, \dot{m}=0.064$ and $a=0.267$. 